\title{
Conversations About COVID Vaccines with Latino Immigrant Communities
}

Julia Koehler MD

The following Do's and Don'ts come from my trial and error process while doing COVID info sessions over Facebook live with immigrant organizations since February 2020. The metrics of my trial and error process are whether there were many questions or more questions than time. These sessions have about 15,000 - 20,000 views at this point; that is why I have the confidence to write down my conclusions on how to do a better job. People have been accepting and kind about my limited language skills; we do not have to be flawless in conducting an info session. Sessions can also be interpreted into the preferred language(s) of participants and still work well.

\section{"Even with native English speakers, it is good to assume no more than elementary school literacy unless the patient indicates otherwise. It is good to assume no numeracy, no statistics, no probabilities, no percent efficacy."}

Studies show that physicians often slip into medical jargon even in conversations with native English speakers and are not intelligible to their patients. Even with native English speakers, it is good to assume no more than elementary school literacy unless the patient indicates otherwise. It is good to assume no numeracy, no statistics, no probabilities, no percent efficacy.

Of note: most people for whom we are holding sessions came to this country because the opportunity to sustain their families at home was not available to them in their place of origin; land, labor, and resources were forcibly taken from their ancestors or themselves. Hence, most had to leave school early, e.g., before the fourth or fifth grade, to help their family survive. Education level in Latin America, like in this country, corresponds most closely to their parent's income. Our conversation partners are smart - they just never had the chance to study biology, chemistry, or math.

These points are for zoom sessions, but many of them can be applied to direct in-person conversations as well.

Do -

- Engage a dialog partner of the organization hosting the event to diminish the lecture-like format that an info session easily turns into.

- Begin with a courteous self-introduction and acknowledgment of the honor and pleasure of being there.

- Speak personally, from the heart.

- Try to look into the camera - on zoom, it then appears like we are looking at the dialog partner or the viewer. It can be very hard to do! - best not to read from a script but to force ourselves to look up at where the camera is. Placing the camera at eye level is perfect. Looking up at it is fine too, looking down at it works less well.

- Speak simply and with understandable metaphors.

- Acknowledge and address people's questions and concerns upfront, before they are explicitly asked. At the same time, every question that gets asked is a good sign for the session going well...

- Be honest about knowns and unknowns and do not try to affirm more certainty than we have. Can say, e.g., Scientists are still studying how much the vaccine protects us from infecting others after we are vaccinated, and right now, we do not know the answer yet exactly. Scientists think that we are less contagious after getting the vaccine, but we are not sure how strong that protection is.

- Frame the decision for or against becoming vaccinated as one that affects not only me personally, but also that I protect my family, my neighbors, my community, and the people I work with by becoming vaccinated because though studies are not complete, it looks like vaccinated people are not going to spread the virus as much as non-vaccinated people, even if they do become infected.

- Highlight that this is everyone's own decision and that no one, including no employer at this time, can force or pressure me to take a vaccine.

\section{"Frame the decision for or against becoming vaccinated as one that affects not only me personally, but also that I protect my family, my neighbors, my community, and the people / work with by becoming vaccinated because though studies are not complete, it looks like vaccinated people are not going to spread the virus as much as non-vaccinated people, even if they do become infected."}

- Start with talking about what a vaccine is. E.g., a vaccine is a way for our body's immune system to go to school and take a lesson. Just like for us, it is good if we had the chance to learn how to make additions in school before we need to add up the prices of what we are buying in a store, it helps our immune system if it has had a chance to have a lesson about a deadly germ before it runs into that deadly germ in real life. A vaccine teaches our immune system by giving it a piece of the germ to study or by giving it a weak or a dead version of the germ. The COVID vaccine contains instructions for our body to make one important piece of the virus, and then our immune system can study that. Just like in school, where we need to study something for a while before we know it 
well, the immune system also needs time. That is why we are not protected until weeks after we get a vaccine. Furthermore, just like in school, where we often need to repeat lessons before we know a subject well, the immune system can often create a better memory of a deadly germ if it gets that lesson more than once. That is why the current COVID vaccines need to be gi

- Talk to the people from the same communities you encounter at work about COVID and vaccines, like security staff, janitors, valets - this provides direct experience with their questions and concerns, and it is good regardless of the pandemic! (It is good to disregard the implicit expectation of intense segregation in this country, where doctors are expected to chat only with doctors and not with janitors. That implicit expectation does not exist in other parts of the globe.)

\section{"Does the vaccine contain a chip by which ICE can localize me? A: no, definitely not; you can look at the syringe with the fluid and see there is no chip in it. Bar codes identify packages with vaccine doses to make theirisposition easy to track; that is the only tracking mechanism."}

\section{- Frequent questions are:}

Does the vaccine contain a chip by which ICE can localize me? A: no, definitely not; you can look at the syringe with the fluid and see there is no chip in it. Bar codes identify packages with vaccine doses to make their disposition easy to track; that is the only tracking mechanism.

What kind of allergies may make it dangerous to take the vaccine - hay fever, allergy to crabs or peanuts, penicillin allergy? A: The risk of a dangerous reaction to the vaccine depends on the kind of allergic reaction we have had in the past. If you have previously had an acute, severe reaction called anaphylaxis that requires you to carry an epi-pen, which is a little pistol that can inject a critical medicine fast, that people carry who have had very dangerous allergic reactions, it is safer to get your vaccine at a health center or hospital where there are doctors who can attend to you if you have a reaction. But everyone is observed for 15 minutes after the injection to watch for allergic reactions.

$\sim$ How safe can these vaccines be after being developed so quickly? A: The technology for mRNA vaccines has been researched for 15 years, so it is not completely new. All of the usual steps were taken, but many were taken at the same time instead of one after the other, in order to speed up the process, given the urgent need in the pandemic. This was possible because lots of money was made available by the government.

$\sim$ Is there a guarantee that there will not be side effects later? A: No, there is no guarantee. Side effects of vaccines usually appear within weeks to months. Millions of people have already received these vaccines, and no common side effects have appeared within the eight weeks they have been given. But we can weigh the risk of later side effects against the risk of contracting COVID. COVID is a dangerous disease that has killed people of all ages, and that has left about a fifth of all who had it struggling with long-term effects like tiredness, body pains, shortness of breath, and damage to the heart, lungs, or kidneys. My risk of contracting COVID in Massachusetts is extremely high. I have to weigh this serious risk of long-term illness and death against my very small risk of experiencing an unknown side effect at some later time. This is the balance I need to consider.

$\sim$ Were cells from aborted fetuses used to make these vaccines? A: No, these vaccines were made synthetically without the use of any human cells. Aborted fetuses' cells played no role in any part of these vaccines. Pope Francis has been vaccinated and has spoken out in favor of receiving these vaccines (https://www. ncronline.org/news/vatican/pope-francis-suggests-people-havemoral-obligation-take-coronavirus-vaccine).

$\sim$ Will these vaccines change my genes, and will I become genetically modified? A: No, because mRNA in the vaccines is instructions for our body that generally come from genes. It is not instructions that go into genes. When you dump color into a stream, that color will show up downstream from where you stand but not upstream. There is no way that the material in vaccines turns into genetic modification.

$\sim$ What is in the vaccines? A: The vaccines contain a piece of instructions for our body to make one little piece of the virus. This piece of instruction for the body is called mRNA. When our body makes this little piece of the virus - never a whole virus - our immune system learns to recognize that piece and starts studying how to defend our body against it. This little piece of instruction, the mRNA, is wrapped into tiny bubbles of fatty material that can enter our body most effectively. These tiny bubbles that have instructions for our body (mRNA) inside of them are in a salt solution like fat in a soup. When we get the vaccine injection, the instruction material enters our system, and our body starts making a tiny, important piece of the virus, but never the whole virus.

$\sim$ Can I get COVID from the vaccines? No, because the vaccines do not contain the virus. However, we might get COVID after becoming vaccinated because we were exposed a few days after we got the vaccine, and our body's immune system did not have time to study the virus before the virus started growing in our body.

$\sim$ My uncle got sick after getting a vaccine. I'm worried I will get sick the same way if I take the vaccine. A: Before the pandemic, many people in Massachusetts got sick and had to go to the hospital. They might have had a stroke, a heart attack, a seizure, a bad infection, or any other number of diseases that had nothing to do with COVID. People will still get these diseases now, during the pandemic. Some people will get them right after being vaccinated because the diseases like heart attacks were already forming in their bodies before they got vaccinated. We cannot blame the vaccines for all these diseases that, unfortunately, people are getting for other reasons. Maybe someone with asthma lives in a polluted area and was going to get an asthma attack anyway, and when they happened to get the vaccine that same day. Or maybe someone smoked for many years and was going to get a heart attack anyway, and they happened to get it the week after they got the vaccine. Still, scientists are carefully studying whether any diseases occur more often after people get vaccinated than they occur in people who have not been vaccinated. 
$\sim$ Can I stop wearing a mask after I have been vaccinated? A: No, because we only know how well these vaccines work when everyone is wearing a mask. We do not know how well they work for people who are not wearing masks, distancing, making sure of good ventilation or air filtration, and keeping their hands clean. Vaccines are just one of the tools that can keep us safe from the virus. But they can be an important tool.

Can undocumented people get a vaccine, and if so, will their information be passed on to ICE? A: Undocumented people can get vaccinated like everyone else. Personal information on vaccinated people will be stored to ensure that everyone gets not just one but two doses. That information will not get passed along to ICE. It is safe for undocumented people to get vaccinated.

$\sim$ Will getting the vaccine involve the "Public Charge" rule that was recently changed by the Trump administration, by which accepting specific benefits from the government makes it more difficult to get a green card or citizenship? A: No, getting vaccinated for free, or using our health insurance card to get vaccinated, will not affect anyone's status under the "Public Charge" rule.

Can I get a vaccine if I have no health insurance? A: Yes. If you have health insurance, bring your insurance information or card to the vaccination site. If you have no insurance, you can get vaccinated without payment.

$\sim$ Should a pregnant woman get vaccinated? A: Pregnant women can get sicker from COVID than if they were not pregnant, and they more often give birth prematurely, which is very bad for the baby. So some experts say pregnant women should get vaccinated. Other experts say they should not because the current vaccines have not yet been tested in pregnant women. So far, no problems were seen for the mother or the baby when the woman got the vaccine before knowing she was pregnant. So this is a decision every mother has to make for herself and talk with her own doctor.

$\sim$ Can a mother who is breastfeeding get vaccinated? A: Yes, because the vaccine contains no virus. It just contains instructions for our body to make a small piece of the virus to train our immune system. So she is not putting the baby at risk of catching the virus when she gets the vaccine.

Can I get the vaccine if I have diabetes, or autoimmune diseases like lupus, or an organ transplant? A: Yes, because the vaccine does not contain any virus. There is no risk of becoming infected from the vaccine. So it is very important actually, if you have any of those conditions, to get the vaccine as soon as possible because it will help train your immune system that may need extra help.

Will a COVID vaccine make me infertile? A: No. No vaccine makes anyone infertile. There is an injectable contraceptive, but it is not a vaccine and does not protect against any infection. If you get the COVID vaccine before you become pregnant, we think you will be better protected against COVID during and after your pregnancy, and your baby will also receive some immunity from you.

Do not -

- Use medical or statistics terms that your grandfather or a relative with no medical or biology knowledge does not understand.

- Sound technical - instead, express medical information in personal terms. E.g., do use "I," "we," "you," to explain a concept.
1. https://www.ncronline.org/news/vatican/pope-francis-suggests-people-have-moral-obligation-take-coronavirus-vaccine

The author has no conflicts to disclose

NT

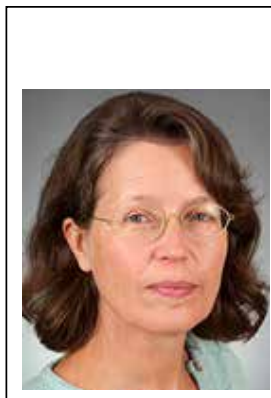

Corresponding Author

Julia Koehler MD

Specialist in Pediatric Infectious Disease

Assistant Professor of Pediatrics,

Harvard Medical School

Boston, Massachusetts

email: decency.matters4us@gmail.com

Readers can also follow NEONATOLOGY

via our Twitter Feed @NEO

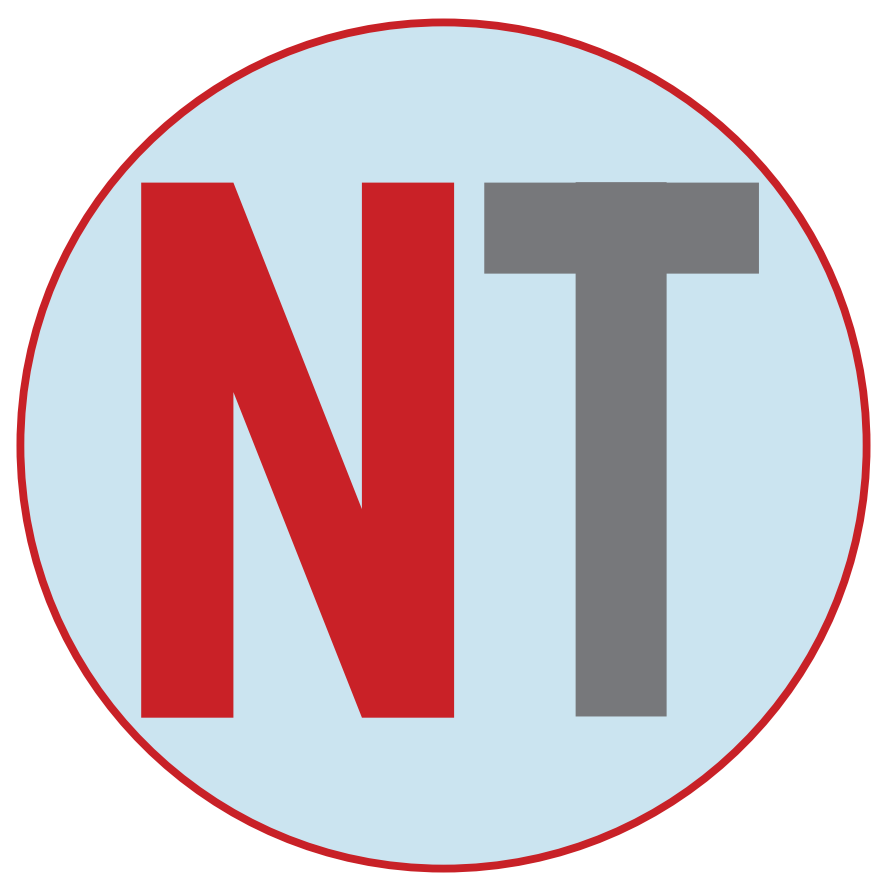

References: 